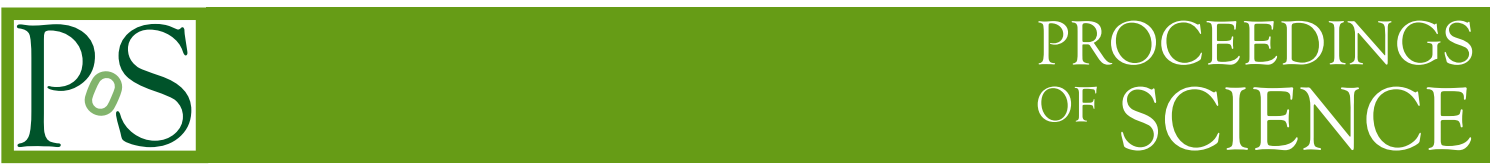

\title{
A charming ICECUBE discover?
}

\author{
D. Fargion, ${ }^{* a b, c}$ P. G. De Sanctis Lucentini, ${ }^{d}$ M. Yu. Khlopov, ${ }^{e, f, g}$ P. Oliva, ${ }^{h, i, c}$ \\ F. La Monaca ${ }^{a}$, P. Paggi ${ }^{a}$
}

a Physics Department Rome University 1, P.le A. Moro 2, 00185, Rome, Italy

${ }^{b}$ INFN Rome1, Rome University 1, P.le A. Moro 2, 00185, Rome, Italy

${ }^{c}$ MIFP, Via Appia Nuova 31, 00040 Marino (Rome), Italy

${ }^{d}$ Physics Department, Gubkin Russian State University (National Research University), 65 Leninsky Prospekt, Moscow, 119991, Russian Federation

${ }^{e}$ Institute of Physics, Southern Federal University Stachki 194, Rostov on Don 344090, Russia

${ }^{f}$ APC laboratory 10, rue Alice Domon et Leonie Duquet 75205, Paris Cedex 13, France

$g$ National Research Nuclear University "MEPHI" (Moscow State Engineering Physics Institute),

31 Kashirskoe chaussee, Moscow 115409, Russia

${ }^{h}$ Niccolò Cusano University, Via Don Carlo Gnocchi 3, 00166 Rome, Italy

${ }^{i}$ Department of Sciences, University Roma Tre, Via Vasca Navale 84, 00146 Rome, Italy

E-mail: daniele.fargion@uniuniromal.it, pietro.oliva@unicusano.it, desanctislucentini.pgegubkin.ru,khlopoveapc.in2p3.fr

Last two years high energy neutrino data are studied. The two recent tau neutrino double bang candidate are discussed within their detectability, noise and expected rate. The neutrino flavor distribution mainly favoring equal electron and muon presence, is reminded. The angular distribution of highest muon neutrino tracks is analyzed. Their horizontal strong anisotropy and their remarkable up-down asymmetry, with the absence of clustering, is noticed. The main persistent missing of astrophysical X,gamma sources (as GRB and AGN flaring source) and all the above signatures led us to suggest a dominance of prompt charmed (atmospheric) events able to pollute, to smear and to hide any minor astronomical presence.

Frontier Research in Astrophysics III, May 2018

${ }^{*}$ Speaker. 


\section{Introduction: ICECUBE Astronomy or a charm neutrino discover?}

The high energy neutrinos up to $\mathrm{TeV}$ are mostly atmospheric, $v^{\mathrm{atm}}$, ruled by an over-abundance of $v_{\mu}$ tracks respect to the cascade showers that are essentially originated by atmospheric $v_{e}$ and neutral current events. The ratio of such $v_{\mu}^{\text {atm }}$ over $v_{e}^{\text {atm }}$ signal is nearly 20:1 [1]. By $v^{\text {atm }}$ we mean the secondary ones from the Cosmic Ray interactions with the atmosphere. Since the Cosmic Rays are charged and bent by the Galactic Magnetic Field, they and their secondary $v^{\text {atm }}$, are smeared and do not allow any astronomy. The data collected by ICECUBE from 2013 shows over 30-60 TeV a remarkable change in the relative abundance of neutrino flavor: $\mathrm{PeV}$ neutrino cascade events have been first discovered [2]. Later on, more data at hundred TeVs confirmed the sudden change from a dominant $v_{\mu}^{\text {atm }}$ flavor at $\mathrm{TeV}$ s energy, to a more dominant cascade neutrino signal with a ratio $1: 3$. These events are just spherical cascades, above tens $\mathrm{TeV}$ up to $\mathrm{PeV}$ [3]. Such neutrino cascades might be made by $v_{e}, v_{\tau}$ and $v_{N C}$ interactions. But also by charmed atmospheric neutrinos: $v_{e}$ and $v_{N C}$. Hereinafter, by $v_{\mathrm{NC}}$ we mean the Neutral Current events made by all of the three flavors. The interpretation for such a flavor revolution [1] has been: Neutrino Astronomy. However, no correlation with nearest or remarkable known (Radio,X, $\gamma$ ) sources and neutrino events arose for the first years, 2010-2017. The poor shower (or cascade) directionality has been forcing us toward a more meaningful astronomy, even made mainly by through-going or crossing induced neutrino muons [4]. Within first several dozens of highest energy ICECUBE events we searched a signal correlation among these sources, [5,6]. More recent analogous attempts have been considered [7]. In September 2017 a rare and unique UHE through-going track event, IceCube-170922A, has been succesfully correlated with a previous high energy gamma Blazar TXC 0506+056, active months earlier as a bright gamma source [8]. This event convinced most authors of the Neutrino Astronomy nature. However, there are several unsolved puzzles to be explained.

Glashow resonance absence. Anti neutrino UHE signals above TeVs exhibit events tracks and cascades, with similar but a little smaller cross sections, in analogy to neutrino ones in ICECUBE. A very peculiar resonant signal by an Ultra High Energy (UHE) anti-neutrino electron at $6.3 \mathrm{PeV}$, may rise with a peak amplified probability, as soon as it is hitting on electron in ICECUBE. These resonant events are making PeV cascade signals. Such an event, called Glashow resonance [9], has not been yet discovered in a contained High Energy Starting Events (HESE) inside ICECUBE. This absence may be a new additional puzzle to be explained, [10]. Nevertheless, one may note that such a partially contained Glashow resonance event has been very recently (ICRC 2017) claimed. The hard astrophysical spectra by exponent -2 or -2.2 for UHE neutrino would be more in disagreement with such paucity. On the contrary a softer charmed atmospheric spectra, with exponent -2.7 or -2.9 will be more consistent with the non observing HESE Glashow resonance.

Flares and GRBs absence. The absence, at high level, of any GRB with several UHE neutrino tracks correlation, the absence of any brightest AGN gamma sources flare in ICECUBE neutrino tracks map, the absence of any Galactic plane-ICECUBE anisotropy, the absence of any self UHE neutrino clustering in narrow solid angles, all of them require an explanation. There is anyway, a very recent, but premature, proposal for a correlation between a blazar NGC1068 source and ICECUBE muon neutrino clustering at TeVs energy [11]. But most or all, the highest UHE alarm event among the last few years remained since a few years uncorrelated with any known active $\gamma, \mathrm{X}$ or radio bright source. For instance the brightest AGN flare (as the huge gamma photon rain [12], on 
3C 279 on June 2015) do not find any UHE neutrino precursor or correlated track partner.

Tau neutrino absence or fewness. The main puzzle is also the absence (or paucity) of any clear $v_{\tau}$ event among several dozens (34) of UHE neutrino cascades above 90-100 TeV. Very recently, since June 2018, ICECUBE offered and claimed two controversial tau candidates [13,14]. It is also to be underlined that among 34 cascades, within our alternative atmospheric charm neutrino scenario, there is anyway room for at least one charmed tau neutrino [15]. In addition to the above absence there is also the average neutrino flavor well consistent with a charm atmospheric one. See Fig.3. Downward muon neutrino track absence. The absence or paucity of downward muon neutrino at hundred TeVs, even respect the upward ones, may be indebt to the ICECUBE Top veto for eventual correlated downward airshower. Consequently, this downward paucity might be explained by an atmospheric neutrino noise veto by the ICECUBE.

\subsection{An Astrophysical hidden origin}

We recall here a cosmological model that propones two populations of galaxies: one is close at a few redshift, while the second is tens redshift far away and somehow able to hide the photon signals due to distance, dust and opacity. Neutrinos from large redshift ( $\mathrm{z}>10)$ are no longer automatically correlated with far but hidden photon signals.

These two ages for the galaxies formation are due to the multi-fluid clustering mechanism in the cosmic expansion [16] and may be related, for instance, to a different helium and hydrogen recombination and to different temperatures while cooling. More exotic reasons for the separate clustering epochs might be also related to the eventual cold dark matter component (as neutrino, neutralino), either by their light or heavy masses, by their different interactions or decouplings [17], via a mixed baryon and dark matter multi-fluid clustering $[16,18,19]$.

The unique event IceCube-170922A observed is at $\mathrm{z}=0.3$. This is in disagreement with the above proposal: a signal without $\gamma$ partners may be expected from the far redshift population and not from the near one.

\section{A possible charm UHE neutrino discover}

A more convincing and testable approach is offered in the present article. It is based on a noisy charm atmospheric component smearing ICECUBE data and hiding the minor astrophysical signals. Many authors agreed that such a charmed atmospheric neutrino component must exist and plays role [20], but most of them considered the charm signals as secondary component, of one third or less of the present observed flux, in the ICECUBE rate. Therefore, most authors, remained convinced of the main UHE neutrino astrophysical nature. We remind anyway that the charmed atmospheric spectra derived by Cosmic Rays, CR, models is not well defined. It is known within a factor two. Moreover, it is dependent on the real, but unknown, PeVs energy CR composition, leaving much room for its eventual underestimated relevance.

\subsection{Sample data and tau events: 2018-2019}

The ICECube event data has not been published in detail for several years. Only maps and energy-coordinate spectra have been released in conferences in 2018-2019. We will use the 103 HESE event data of 2018 [21,22], see Fig.1, and the most updated UHE neutrino map [7] of 2019, 


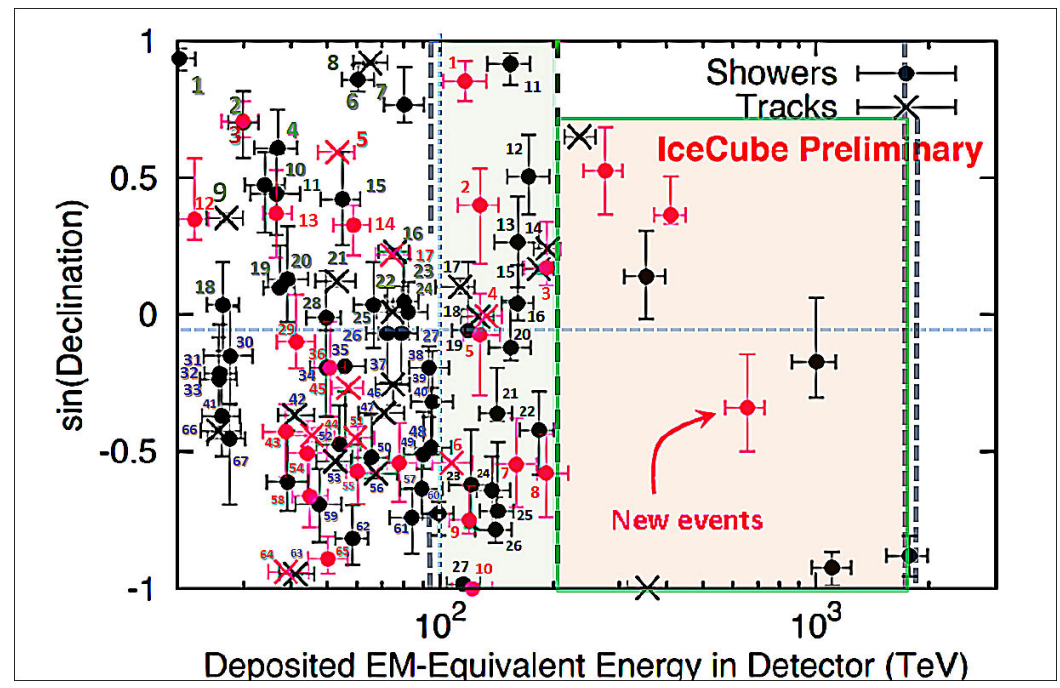

Figure 1: The last (June 2018) 103 HESE data set [21,22] On the vertical axis the HESE events arrival direction, in $\sin ($ Declination) within celestial coordinate, while on the horizontal one the deposited EMequivalent energy released in the detector. The energy lower bound threshold has been reduced to $100 \mathrm{TeV}$, see dotted line (or even at $90 \mathrm{TeV}$ as in the colored areas); the two $\tau$ candidates to inspect are just among 27 events above $100 \mathrm{TeV}$ and 9 above $200 \mathrm{TeV}$, for a total of 36 events. Adding the events in the range 90-100 TeV the total number rises to 42 . Among them we may observe 34 shower that might be made not only by an $v_{e}$ and more rarely by a neutral current, but also by $v_{\tau}$, on average nearly a dozen. On the extreme sides of the dectection range: the highest energetic shower at $2 \mathrm{PeV}$ and a weak event at $89 \mathrm{TeV}$ are the two Tau shower candidates offered by ICECUBE.

see Fig.4, where we also combined the early information on the UHE HESE ICECUBE neutrino events shown in [21,22], to disentangle external tracks (the through-going) from the internal HESE ones, see Fig.1 and Tab.1. We can distinguish events of three types: first the High Energy Starting Events, or HESE, tracks originated inside the ICECUBE detection volume, second the HESE cascades originated and completely contained in the same volume, and third the tracks not HESE, but through-going, originated outside and crossing the whole detection volume. We can count 26 HESE tracks, 76 HESE cascades, for an overall 102 HESE events and nearly 60 . The total track number, HESE and external, is 86 as shown in Fig.4. Even if they were originally presented togheter [7], we distinguish them via the colour label, as shown in Fig.4 and explained in the relative caption. We also maked in the Fig.1 the energy ranges associated to the two tau candidate event claimed by ICECUBE at $89 \mathrm{TeV}$ and $2 \mathrm{PeV}[13,14]$.The correlated Fig. 2 shows the corresponding ICECUBE energy ranges in function of the detection ability and probability [23].It is easy to underline the low probability to be both of them real tau signal events. For instance at lowest energy, at $(80+9)$ $\mathrm{TeV}$ one, the expected atmospheric neutrino noises exceeds by a factor four any astrophysical signal [23]. The consequent tau track at $90 \mathrm{TeV}$ would be expected at around 4.5 meters, and not at the 17 observed meters. Indeed, the observed first bang energy released by the $2 \mathrm{PeV}$ event is much larger respect to the second one. The theoretical tau double bang should show an opposite energy imprint: first a small and later a larger second shower, twice times or more brighter [24]. Moreover, because $L_{\tau}=49\left(E_{\tau} / \mathrm{PeV}\right)$ meters, the tau distance at $2 \mathrm{PeVs}$ should range around hundred meters 


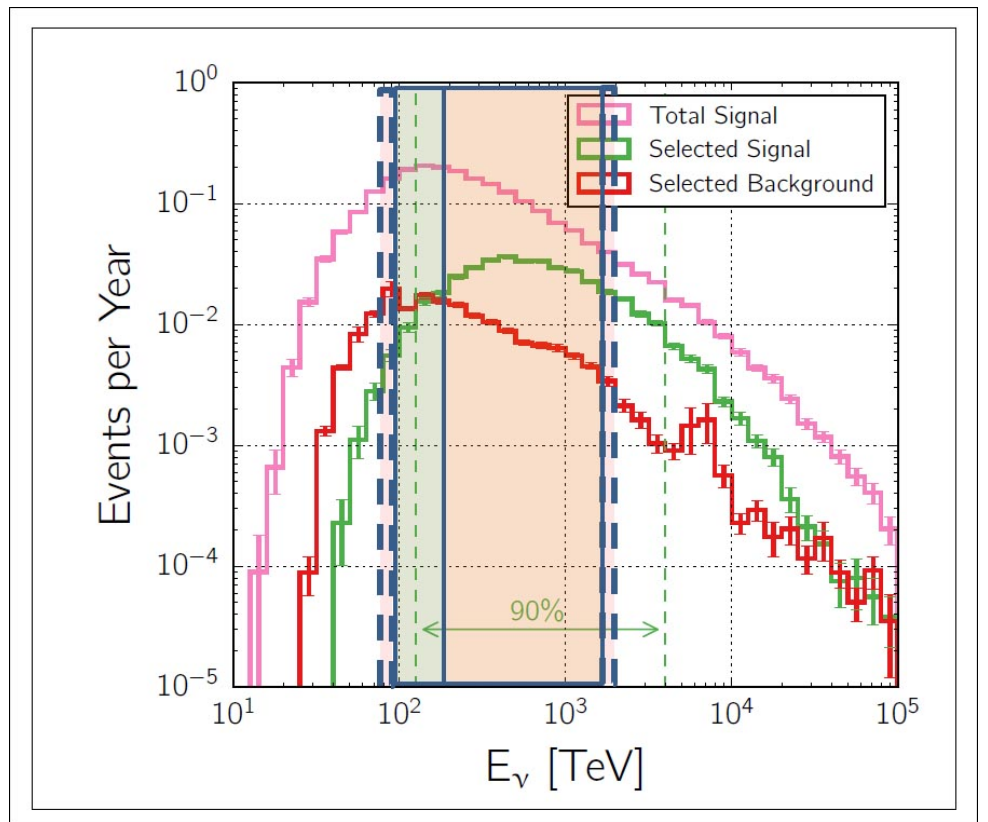

Figure 2: The ICECUBE expected event rate of Tau neutrino as a function of the energy for signals (green curve) and noises (red curve). Note that the lowest energy tau event, at $89 \mathrm{TeV}$, is the most un-probable signal. Noise is four times larger than signal. Note also that the highest Tau event at $2 \mathrm{PeV}$ it was revealed on 2014. In (opposite) analogy a lowest $89 \mathrm{TeV}$ signal would track mostly around 4.5 meters, while the observed double bang for the weakest event is this time four times longer, around 17 meters. The colored rectangular areas (on the left, the most crowded one, on the right, the most probable one) are surprisingly empty of any tau events.

and not just the 16 meters as the observed one.

\subsection{Tau neutrino as a probe of Neutrino Astronomy}

Its presence as abundant as the other flavours $v_{\mu}$ and $v_{e}$ will clearly confirm the astrophysical origin. This full flavor mixing is guaranteed even by the tiny neutrino masses and by their splitting and mixing at the atmospheric and solar observed levels. Indeed, the astrophysical neutrinos are mostly oscillated and mixed along the widest stellar and cosmic flights, leading in most scenarios to a complete averaged equal three flavor $v_{\tau}, v_{\mu}, v_{e}$ probability. Therefore, $v_{\tau}$ might and should be present and possibly observable in the several ICECUBE events above hundred TeV. This is not yet (well) observed. The UHE muon neutrino $v_{\mu}$ are easely produced by pion and Kaon decay in our atmosphere up to TeVs. Instead, their secondary energetic $v_{e}$ are hardly produced by the muon decay in-flight. Their $v_{\tau}$ component is absent, even by the $v_{\mu}$ oscillation along the Earth. Moreover, the UHE $v_{\tau}$ are negligible (a tenth of muon ones) even in the charmed atmospheric component because they are suppressed by a smaller cross-section for the charmed atmospheric $v_{\tau}$ component [25]. Therefore, an evident tau presence will mark the astrophysical origin of the events. This explains the importance of the tau role, a key role, and clarifies our persistence in seeking the definitive discovery of the tau neutrino [26,27]. 


\subsection{Tau neutrino detection}

How could UHE $v_{\tau}$ be detected in ICECUBE? A UHE $v_{e}$ with energy in the range from several $\mathrm{TeVs}$ up to $\mathrm{PeV}$, traveling through the detector volume, interacts with the nuclei creating a shower or cascade: several meters of tree rich ramification in pions and electromagnetic secondaries; the secondaries Cherenkov optical photons of this cascade will be diffused in ice by random walk into a spherical shape: a cascade. For a shower of hundreds $\mathrm{TeV}$ the size of the sphere can reach up to hundreds meters. Largest $\mathrm{PeV}$ cascade events may extend their Cherenkov lightening in several hundreds meter spherical radius, filling most of the detector volume. By the timing spread or diffusion in it is possible to estimate somehow the neutrino directionality within a wide solid angle ( $\sim \pi \theta^{2}$ with $\left.\theta=(15 \text { to } 30)^{\circ}\right)$. In general, also the Neutral Current and the relevant tau by $v_{\tau}$ event at tens $\mathrm{TeV}$ may lead to a similar cascade as the $v_{e}$ one. Indeed, low energy tau decay overlaps its birth place hiding the short track inside the light bang. There are observable different signatures, as discussed soon, for more energetic $v_{\tau}$ events above hundred TeV. Therefore, in ICECUBE present resolution, UHE neutrino cascades up to nearly hundred TeVs are well hiding their own neutrino flavor identity. However, above the hundred $\mathrm{TeV}$ threshold the $\tau$ fast decay $\left(2.9 \cdot 10^{-13} \mathrm{sec}\right.$.) may be separated and resolved by their $v_{\tau}$ birth cascade, both in distance and in time. This occurs because the $\tau$ relativistic boosted life is leading to two separated cascades, two bangs, linked by 49 $\cdot\left(E_{\tau} / \mathrm{PeV}\right)$ meters track size [24]: a first UHE $v_{\tau}$ hadron nuclear interaction with a fourth or a third of the primary $v_{\tau}$ energy; then a later $\tau$ decay with the rest major part of the $v_{\tau}$ energy. These two bangs may be both contained in the present $\mathrm{km}^{3}$ detector volume. Because of the actual detector size and the optical array resolution, the best detection energy window for such $\tau$ double bang is with tracks from tens to hundreds meters: an energy within a range of nearly $100 \mathrm{TeV}$ up to a few or several PeV, as shown in the Fig.2. We note the maximal probability to detect such a double bang [23], occurred around $400 \mathrm{TeV}$, quite distant from both from the $89 \mathrm{TeV}$ and $2 \mathrm{PeV}$ observed events at the extreme edges shown in Fig.2.

\subsection{Tau neutrino airshower}

An analogous more filtered $v_{\tau}$ signal would be an UHE PeV-EeV up-going tau escaping from a mountain or the Earth airshowering to the sky. In synthesis the double bang [24] considered above in ICECUBE occurs with a first bang inside the mountain or inside the Earth crust, and the second bang in the air. This consequent tau airshower Astronomy has been offered since nearly 20 years $[26,28]$. Unlike isolated upward muon tracks, the upward tau airshower expands in a vast area (km square size) its presence, by a million or even thousand of billion secondary traces. The event is an ideal filter and amplifier of Neutrino Astronomy. Tau airshower has been studied in more detail in flight across the Earth opacity [27]. It became more and more experimented only in the recent decade. The upward tau airshower have not yet been observed. This tau airshower signal, often referred by an improper name as a skimming neutrino [29], could and should offer the ideal noisy free Neutrino Astronomy. Several present and on-going experiment are searching for such tau airshowers upgoing from the Earth toward the horizontal sky. AUGER or TA array UHECR records by fluorescence lights, by upgoing horizontal airshower, might soon reveal such $\mathrm{EeV}$ neutrino traces. 


\subsection{ICECUBE neutrino flavors}

Up to early 2018 Icecube claimed the 102 HESE events, 76 cascades and 26 tracks, all above $20 \mathrm{TeV}$ energy, with no $\tau$ detection. Since June 2018 two $\tau$ event candidates have been finally claimed. However, as we shall argue, both of them are not a probable tau candidate signal because of the disagreement between their observed versus expected $\tau$ signature. Therefore, tau neutrino signals among last ICECUBE HESE 76 cascades, in particular the most energetic ones above 100 $\mathrm{TeV}$ ( 28 cascades), or above $90 \mathrm{TeV}$ (34 cascades), should contain nearly a dozen of $\tau$ double bang. The only two observed events are too few respect the predictions: the lowest energy one is located in a range of energies where is polluted by too much noise and the second is distributing the energy between the two showers in a way opposed to the expectations.

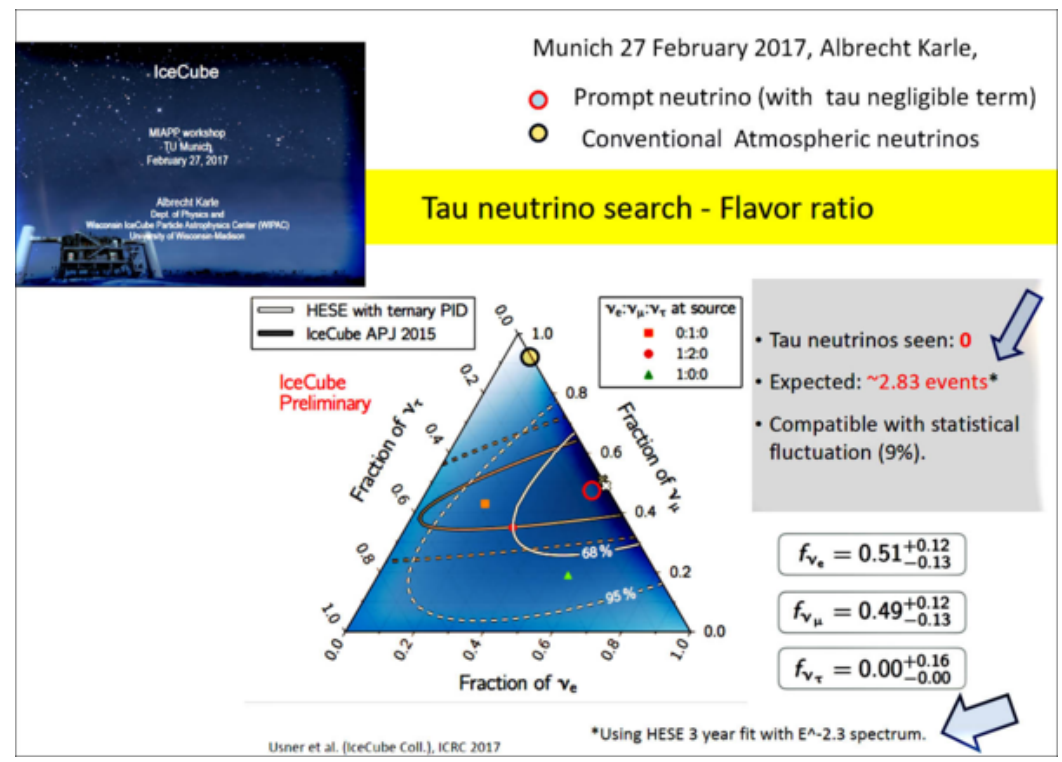

Figure 3: Elaboration of Fig.4 from [30]. A recent article based on 2017 record by ICECUBE has been favoring not the common expected astrophysical flavor ratio $1: 1: 1$ but a flavor combination tuned with the charmed case: $\phi_{v_{\mu}}=\phi_{v_{e}}$. More exactely: $\phi_{v_{\mu}}=0.49 \%$ and $\phi_{v_{e}}=0.51 \%$. The blue ring on the top shows the muon atmospheric dominated role with a tiny $5 \%$ component of the electron ones. The presence of a small tau component also at $5 \%$ ratio, occurs even for the atmospheric charmed neutrino component, as for the case of one or two tau labeled by a red ring that is well correlated with the white cross of the observed data. It should be noted also the expected tau event rate of 2.83 event every three years is well comparable with the expected 9 events discussed in our article for the whole 2011-2019 period [15].

Without the two tau events in 2018 one may inspect the whole ICECUBE neutrino map in a flavor triangle map. For instance the weighted map of 2017 by ICECUBE, see Fig.3. One may observe that the most probable flavor point for an astrophysical model (red dot) is near the center by an equipartition flavor ratio $: v_{e}: v_{\mu}: v_{\tau}=1: 1: 1$. The most probable observed signal (dense area) is more centered on a very different flavor side: $v_{e}: v_{\mu}: v_{\tau}=1: 1: 0$. This is just the expected charmed atmospheric noise that we are here defending. The tiny component of an eventual charmed tau component will lead to a similar ratio: $v_{e}: v_{\mu}: v_{\tau}=1: 1: 0.05$, as shown by a red ring in the Fig.3. Naturally the new two tau events in 2018 may partially correct the flavor weight in favor of an 
astrophysical component. Nevertheless, the two mentioned tau events are not both ideal convincing signals. Moreover, in the same ICECUBE in 2017 presentation, see Fig.3 it was foreseen a tau rate event of nearly three event every three years, leading to almost nine event today. In agreement with our first estimate, in disagreement with the too few and improbable two tau events.

\section{Upward-Downward tracks anisotropy and asymmetry}

A very recent August 2019 ICECUBE map shown in ICRC, is containing more useful updated information. There are hundreds of recent UHECR events (by AUGER and TA, Telescope Array), all the ICECUBE cascade HESE events (76 events) and muon HESE neutrino tracks (26), reaching nearly 86 total tracks, see Fig.4. In this figure we overlap the HESE muon tracks with 2018 map data $[7,21]$. This comparision permited us to disentangle through-going muon tracks (by UHE neutrino, crossing ICECUBE from side to side) from HESE contained muon tracks. This disentanglement is shown by differnt colors for the event numbers in Fig.4. We grouped the events in following ranges of zenith angles above and below the horizon: above $(0 \text { to } 15)^{\circ},(15 \text { to } 30)^{\circ},(30$ to 45$)^{\circ},(45 \text { to } 60)^{\circ}$ and $(60 \text { to } 90)^{\circ}$; and below $(0 \text { to }-15)^{\circ},(-15 \text { to }-30)^{\circ},(-30 \text { to }-45)^{\circ},(-45$ to -60$)^{\circ},(-60 \text { to }-90)^{\circ}$. About the upward tracks we colored in red the HESE ones and in green the through-going ones. About the down-ward tracks we used the black for the HESE and the blue for the through-going ones. The track resolution is within $1^{\circ}$ and allows us to discuss some remarkable angular anisotropy and asymmetry for tracks. We made similar count for the cascades: because of their wider and smeared angular resolution, about $15^{\circ}-30^{\circ}$, we classify them just for the sign of the arrival direction in the upward and and in the down-ward groups. We analized these events in the view of an astrophysical or an atmospheric charmed nature deriving the correponding expected rate and anisotropy and asymmetry.

Let us remind here that by upward event we mean a signal from North sky and by downward event a signal coming from the South sky. The straightforward message that arises in the cascades and tracks map is the opposite up-down ratio. There are 29 upward and 47 downward cascades. Their ratio is $R_{D o w n / U p}^{c}=47 / 29=1.62$. On the contrary, there are 57 upward tracks (HESE and through-going) and 29 downward ones. Their ratio is $R_{\text {Down } / U p}^{t}=0.5089\left(R_{U p / \text { Down }}^{t} \simeq 1.965\right)$. To be underlined the opposite cascade (poorer upward) and tracks (poorer downwards) ratio signature. Their ratio $R_{\text {Down } / U p}^{c} / R_{\text {Down } / U p}^{t} \geq 3.18$ has different key explanations.

The upward cascade remarkable asymmetry $(\mathrm{R}=1.62)$ and its paucity might be related mainly to the Earth opacity for UHE neutrinos. Indeed, the HESE tracks show a negligible asymmetry $R_{\text {Down } / U p}^{t(H E S E)}=14 / 13=1.07$, far from the cascade ones, $\mathrm{R}=1.62$. It might be due to a competitive detection efficency veto by downward atmospheric noise. Tracks by through-going muons, of external events, are quite long outside the detector, as we will estimate later nearly $2.5 \mathrm{~km}$. The 1.45 kilometer thick ice layer above the detector makes less probable the downward through-going muons signals compared to the upgoing UHE ones. But Earth opacity might also compensate this effect. Upward through-going tracks below $-34^{\circ}$ are coming mostly interacting inside the rock Earth, where is the main calorimeter. The same Earth opacity for through-going UHE neutrino plays an opposite relevant role, but it is overcome by previous efficency. They are 44 upward and 15 down-ward $R_{\text {Down } / U p}^{t(\text { Throug })}=0.341$. This very strong asymmetry respect cascades is due to $v_{\mu}$ interaction probability, to their lenght in ice and rock and to the strong downward veto used to 
exclude the atmospheric noise: when a downward muon has a correlation in time and direction with an an atmospheric event, it can fall under the veto triggered by the Ice Top CR array. It may also rarely happen that a through-going track comes together with a twin charmed companion and finish to be discarded as atmospheric noise. Cascade are so much smeared in angular direction that their eventual atmospheric shower and origination avoid the IceTop veto. Moreover, there is no UHE

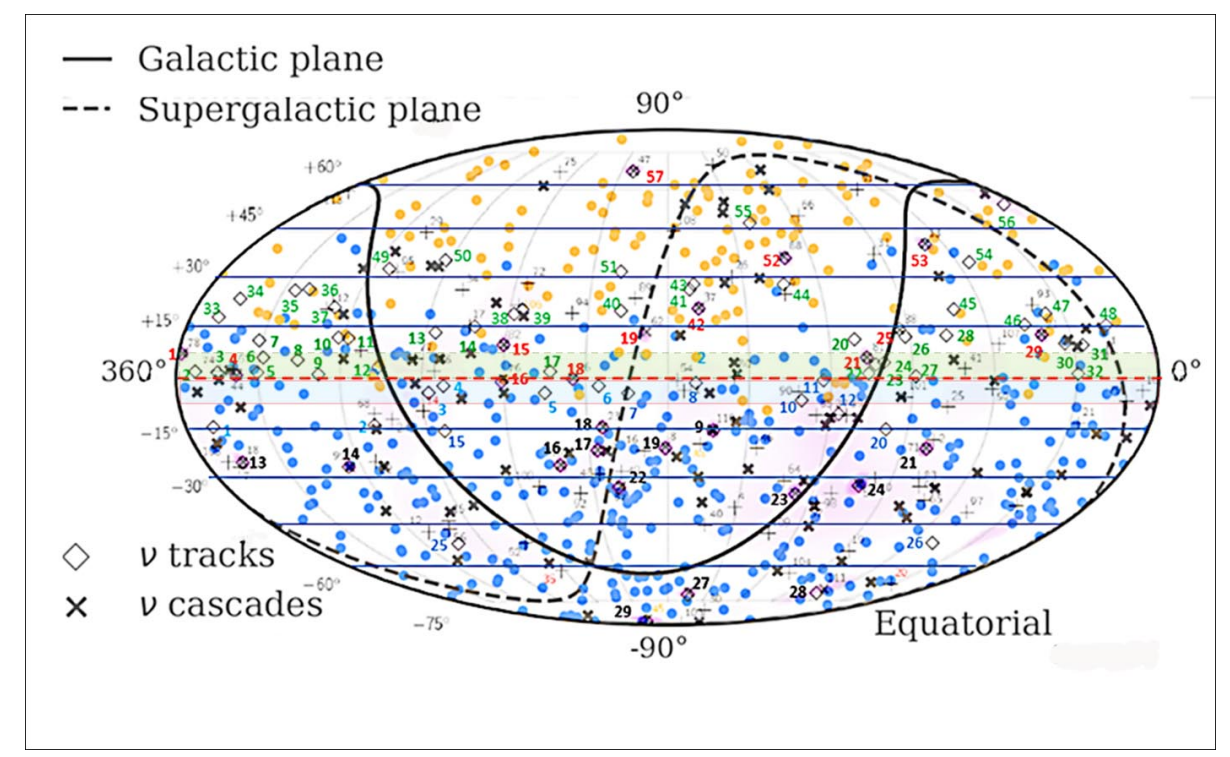

Figure 4: The most recent ICECUBE event map, combined with several collaborations shown in ICRC 2019 [7]. ANTARES contributed by 3 events. AUGER and TA (Telescope Array) UHECR events are labeled respectively by blue and orange disks. The $v$ events are both originated by $v_{\mu}$ leading to tracks (a diamond symbol) or a $v$ cascades, labeled by a dark cross, made possible by either a $v_{e}, v_{N C}$ or by $v_{\tau}$ UHE neutrino interaction. The $v_{\mu}$ tracks themseles might be originated either inside the ICECUBE named HESE (high Energy Starting Event) or they are made by through-going muons whose $v_{\mu}$ interaction and birth it is outside the IceCube $\mathrm{km}^{3}$ itself. These through-going muons tracks are disentangled by the overlap of ICRC 2019 [7] by HESE events map 2018 [21]. The cascade events are much smeared in their detection. Therefore, they are counted only in widest solid angle, just to verify the upward downward ratio. The HESE track event presence is marked by an underlined violet mini cross over (the otherwise) empty diamond. Remaining empty diamonds are just labeling through-going muon tracks born outside the $\mathrm{km}^{3}$ detector. We pointed the upward (from the North sky) tracks by numbering them in horizontal lines among sequence of $(0 \text { to } 15)^{\circ},(15 \text { to } 30)^{\circ},(30$ to 45$)^{\circ},(45 \text { to } 60)^{\circ}$ and $(60 \text { to } 90)^{\circ}$ solid angles. The Upward tracks are label by the red colour (HESE) number while green colour numbers are the through-going muons events. In analogy the downward (from South Pole Sky) tracks are counted in $(0 \text { to }-15)^{\circ},(-15 \text { to }-30)^{\circ},(-30$ to $-45)^{\circ},(-45 \text { to }-60)^{\circ},(-60 \text { to }-90)^{\circ}$ lines. The black numbers label the HESE contained events while the blue numbers are pointing to through-going muons. The dashed red line describe the IceCube horizons in celestial coordinate. As in the text the whole sample is strongly anisotropic, enhanced at horizons. Moreover there is an additional asymmetry among horizontal upward (more abundant) and downward (less events) tracks. In the text the main consequences. 
electron charm patners able to come inside the detector. Therefore, such downward cascade are in proportion more abundant than the downward tracks. This asymmetry for contained HESE events already suggested the atmospheric polluted dominant role. Just assuming a symmetric up-down ratio to satisfy the HESE tracks, with 47 downward cascades versus 29 upward ones, the asymmetry will be statistically quite unprobable: by a binomial probability calculations it will be below $1.1 \%$. This probability is indeed mitigated for the observed ratio $R_{D o w n / U p}^{t(H E S E)}=14 / 13: P=2 \%$. We already mentioned that the through-going muon neutrino tracks are long and exceed the ICECUBE size crossing the detector side to side. This de facto increases the observable neutrino volume. The increase, being only longitudinal, grows linearly with the muon characteristic length. At hundreds of $\mathrm{TeV}$ the muon track in the ice is less than ten kilometers long due to energy losses [26] The search of selected UHE astrophysical neutrinos at hundreds $\mathrm{TeV}$ makes them originated mostly nearer than $10 \mathrm{~km}$ from the detector [26, 27]. We may reach a first average estimate of the muon track outside the ICECUBE assuming in a first approximation the ratio of the through-going event respect to the HESE tracks of recent 7 years: 59 through-going events vs 26 HESE, with a ratio of 2.26. Hence, we may assume that the average muon tracks outside the detector should be about 2.2 $\mathrm{Km}$ long. An additional estimate may be reached by similar ratio found in the horizontal angular strip of $0^{\circ}-15^{\circ}: 23$ through-going events versus $9 \mathrm{HESE}$, leading to 2.55 ratio. In conclusions the muon tracks outside ICECUBE are on average well below three kilometers long, let us assume for the following calculations $L_{\text {ext }}=2.5 \mathrm{~km}$. The solid angle at $\pm 15^{\circ}$ above and below the horizon in first approximation insists on a comparable slanth depth. This would be true for a detector located at least $0.65 \mathrm{~km}$ over the ice. However, the detector sits just at 370 meters from the rock. Therefore, a tiny component of the detector volume may be recording UHE neutrinos produced also in the rock, partially breaking the up-down symmetry. The much narrow solid angle of $\pm 7.5^{\circ}$ guarantees that ICECUBE is observing comparable slanth depth in ice above and below the horizon. The $\pm 7.5^{\circ}$ solid angle contains 25 events, as shown in Fig.4 and reported in Tab.1: $(0 \text { to } 7.5)^{\circ}$ with 17 upward events, and $(-7.5 \text { to } 0)^{\circ}$ with 8 downward. The probability $P$ that this asymmetry up-down occurs, assuming as we said a symmetric astrophysical signal, is near to $P=3.22 \cdot 10^{-2}$. In the more statistical populated range $\pm 15^{\circ}$ (with a tiny almost negligible pollution rate from the the rock) the consequent probability due of the larger numbers (32 upward versus 12 downwards) is:

$$
P=1.19 \cdot 10^{-3} \text {. }
$$

All these are additional hints that there is a peculiar veto for downward tracks related to the ICE TOP detection of polluted airshowers. This estimate may be extended to arrival solid angle as large as $\pm 30^{\circ}$, with some cautions because of the Earth opacity that may however suppress, but not increase, the upward versus downward track ratio. The underground rock increases with relevance the upward rate. The two effects may not always be comparable and may not mutually wipe out. The upward tracks (48) versus the downward ones (21) imply a probability to occur as small as:

$$
P=4.57 \cdot 10^{-4} \text {. }
$$

The analogous estimate for similar upward (38) and downward (13) asimmetry for through-going tracks may occur only for:

$$
P=2.11 \cdot 10^{-4}
$$


These last two probabilities might be considered with much caution than the previous ones. What stated above may strongly suggest a relevant atmospheric veto for the downward events due to their major atmospheric nature, veto absent instead for the upward events coming across the Earth from the North sky.

One may be tempted to suggest a dominant common atmospheric origin via Kaon and Pion to explain the overabundance of the horizontal signals. Indeed, for the ideal angular distribution in spherical symmetry, we may count overabundance in horizontal solid angle ranges of $0^{\circ}-15^{\circ}$ that insists over a solid angle fraction of the sky (over the whole $4 \pi$ ), $\sin \left(15^{\circ}\right) / 2=0.1294$. The probability to find 32 tracks inside such a narrow upward solid angle of $0^{\circ}-15^{\circ}$ within 86 tracks is:

$$
P=8.57 \cdot 10^{-9} \text {. }
$$

This horizontal track overabundance cannot be indebt to largest decay distances for skimming pions and Kaons at far horizons edges: indeed $v_{e}$ may be generated by Kaons and Pions with a suppressed ratio respect to $v_{\mu}$ (of $\sim 5-10 \%$ ) in disagreement with the superior abundance of the recorded cascades versus muon tracks. Therefore, the charmed atmospheric role is the fitting one leading as we mentioned to cascade event number more numerous than tracks, see Fig.3. There is also an independent tool based on the asymmetry among upward and downward cascades and tracks above $80 \mathrm{TeV}$. As shown in Fig 1 the upward HESE tracks are 6 while the upward cascades are 10. Their ratio is $R=(10 / 6)=1.666$. The downward cascades above $80 \mathrm{TeV}$ are nearly 24 while the down tracks are 2 . Their ratio is $R=(24 / 2)=12$, in deep contrast with previous one $R=1.666$. Assuming as true the upgoing ratio $\mathrm{R}=1.666$, the consequent downward configuration binomial probability to occur becomes:

$$
P=\frac{26 !}{24 ! \cdot 2 !} \cdot\left(\frac{10}{16}\right)^{24} \cdot\left(\frac{6}{16}\right)^{2}=5.7 \cdot 10^{-4} .
$$

A quite unprobable rate of events: too few downward muon tracks or too many downward cascades. Only an atmospheric veto may lead to such a paucity of the muon downward tracks. To make the cascade and muon rate comparable in up and down sky we need nearly 14.4 muons versus the observed 24 cascades. The observed tracks are instead just 2. As a first preliminary estimate the possible polluted signals exceeds by a factor 7.2 the eventual astrophysical ones. This may mean nearly $13.9 \%$ of astrophysical signals in a charmed ruling sky. Therefore, for us the natural explanation remains the charm atmospheric noise signal able to hide most of the Neutrino Astronomy in a smeared track sky.

\section{Conclusions}

Any astrophysical neutrino origin assumption should lead to a democratic flavor ratio $1: 1: 1$ : (1) for $v_{e}: v_{\mu}: v_{\tau}:(\mathrm{NC})$ not yet observed and statistically unlikely with the available data. One would expect a dozen of such $\tau$ signals. Two $\tau$ candidates are too few events, both of them in an unprobable energy windows. A charmed atmospheric spctra with an exponent power $-2.7-2.9$ may better hide the eventual Glashow resonance signal and its present absence. The abundance of upwards vs downwards track events might be the result of some anticoincidence trigger selection procedure, that made the veto of a downward atmospheric signal. Implying their terrestrial 


\begin{tabular}{|c|c|c|c|c|c|c|c|c|}
\hline & \multirow{2}{*}{ Ranges } & \multicolumn{4}{|c|}{ Tracks } & \multicolumn{2}{|c|}{ Cascades } & \multirow{2}{*}{ All } \\
\hline & & All & HESE & T-G & Sum & Group & Sum & \\
\hline \multirow{5}{*}{ 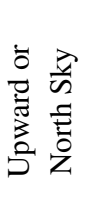 } & $(60 \text { to } 90)^{\circ}$ & 1 & 1 & 0 & ) & 2 & \multirow{5}{*}{29} & \multirow{10}{*}{76} \\
\hline & $(45 \text { to } 60)^{\circ}$ & 2 & 0 & 2 & & 10 & & \\
\hline & $(30 \text { to } 45)^{\circ}$ & 6 & 2 & 4 & 57 & 10 & & \\
\hline & $(15 \text { to } 30)^{\circ}$ & 16 & 1 & 15 & & 17 & & \\
\hline & $(0 \text { to } 15)^{\circ}$ & 32 & 9 & 23 & & 17 & & \\
\hline \multirow{5}{*}{ 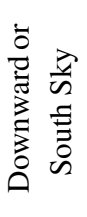 } & $(0 \text { to }-15)^{\circ}$ & 12 & 1 & 11 & \multirow{5}{*}{29} & 28 & \multirow{5}{*}{47} & \\
\hline & $(-15 \text { to }-30)^{\circ}$ & 9 & 7 & 2 & & 28 & & \\
\hline & $(-30 \text { to }-45)^{\circ}$ & 3 & 3 & 0 & & 15 & & \\
\hline & $(-45 \text { to }-60)^{\circ}$ & 2 & 0 & 2 & & 12 & & \\
\hline & $(-60 \text { to }-90)^{\circ}$ & 3 & 3 & 0 & & \} 4 & & \\
\hline
\end{tabular}

Table 1: The present Table is derived from the Fig 4. The tracks on the left side and the cascades on the right side are counted in their different zenith angle views. For sake of simplicity we recall here the counting for a couple of more narrow ranges: $(0 \text { to } 7.5)^{\circ}$ with 17 upward events, and $(-7.5 \text { to } 0)^{\circ}$ with 8 downward.

origination. In this view we may also understand the asymmetry among cascades and tracks ratios in upward and downward sky, see in Eq.3.5: a very unprobable one, about $5.7 \cdot 10^{-4}$. Our present explanation for this small amazing ratio is the suppression made by a veto hiding downward muon tracks. The veto is also adding evidences of the main atmospheric charmed nature for the downward muon tracks. A more complete and accurate data set is needed to refine the calculation goodness for the assumption of the astrophysical vs atmospheric origin. This charm presence may explain at once the persistent puzzling absence of $\gamma-v$ correlations, the absence of narrow neutrino clustering and the missing of the galactic plane signature.

\section{Acknowledgements}

The authors would like to thank for the suggestions the Prof. Barbara Mele and Aleandro Nisati for the estimate of the radiative corrections in the Glashow diagrams.

The work by M. Yu. Khlopov was supported by grant of the Russian Science Foundation (project No-18-12-00213).

\section{References}

[1] D. Fargion and P. Paggi, Flavor revolution at ICECUBE horizons?, Nucl. Instrum. Meth. A753 (2014) 9 [1310.3543].

[2] IceCube Collaboration collaboration, M. G. Aartsen, R. Abbasi, Y. Abdou, M. Ackermann, J. Adams, J. A. Aguilar et al., First observation of pev-energy neutrinos with icecube, Phys. Rev. Lett. 111 (2013) 021103.

[3] IceCube Collaboration collaboration, M. G. Aartsen, M. Ackermann, J. Adams, J. A. Aguilar, M. Ahlers, M. Ahrens et al., Observation of high-energy astrophysical neutrinos in three years of icecube data, Phys. Rev. Lett. 113 (2014) 101101.

[4] D. Fargion, P. Oliva and P. G. De Sanctis Lucentini, Crossing muons in icecube at highest energy: a cornerstone to v astronomy, Nuclear Physics B - Proceedings Supplements 256-257 (2014) 213. 
[5] D. Fargion, G. Ucci, P. Oliva and P. G. De Sanctis Lucentini, The meaning of the UHECR Hot Spots: A Light Nuclei Nearby Astronomy, EPJ Web Conf. 99 (2015) 08002 [1412.1573].

[6] D. Fargion, P. Oliva, P. G. D. S. Lucentini, D. D’Armiento and P. Paggi, Uhecr narrow clustering correlating icecube through-going muons, Nuclear and Particle Physics Proceedings 291-293 (2017) 195 .

[7] A. Barbano, Search for correlations of high-energy neutrinos and ultra-high energy cosmic rays, in 36th International Cosmic Ray Conference (ICRC2019), vol. 36, 2019, https://pos.sissa.it/358/842/pdf.

[8] I. Collaboration, Neutrino emission from the direction of the blazar txs 0506+056 prior to the icecube-170922a alert, Science 361 (2018) 147

[https://science.sciencemag.org/content/361/6398/147.full.pdf].

[9] S. L. Glashow, Resonant Scattering of Antineutrinos, Phys. Rev. 118 (1960) 316.

[10] S. Sahu and B. Zhang, On the non-detection of glashow resonance in icecube, Journal of High Energy Astrophysics 18 (2018) 1 .

[11] ICECUBE collaboration, M. G. Aartsen et al., Time-integrated Neutrino Source Searches with 10 years of IceCube Data, 1910.08488.

[12] D. Fargion and P. Oliva, Fast 3c 279 gamma flares by a merging medium size black hole jet aligned to the agn one by tidal torque, in XI Multifrequency Behaviour of High Energy Cosmic Sources Workshop, vol. 246, p. 053, SISSA Medialab, 08, 2016.

[13] J. Stachurska, New measurement of the flavor composition of high-energy neutrino events with contained vertices in icecube, in Poster at XXVIII International Conference on Neutrino Physics and Astrophysics, 4-9 June 2018, Heidelberg, Germany, Zenodo, 2018, DOI.

[14] ICECUBE collaboration, J. Stachurska, First Double Cascade Tau Neutrino Candidates in IceCube and a New Measurement of the Flavor Composition, in HAWC Contributions to the 36th International Cosmic Ray Conference (ICRC2019), 2019, 1908.05506.

[15] D. Fargion, P. G. De Sanctis Lucentini, M. Yu. Khlopov, P. Oliva, F. La Monaca and P. Paggi, No guaranteed neutrino astronomy without (enough) double bang tau and downward HESE muon tracks, arXive-prints (2018) [1808.10493v4].

[16] D. Fargion, Gravitational instability for a multifluid medium in an expanding universe, Il Nuovo Cimento B (1971-1996) 77 (1983) 111.

[17] F. Antonelli, E. Konoplich and D. Fargion, Right-handed neutrino interactions in the early universe, Lettere al Nuovo Cimento (1971-1985) 32 (1981) 289.

[18] D. Fargion, Right-handed and left-handed neutrinos and the two galactic populations of the universe additional evidence for the neutrino mass, Il Nuovo Cimento B (1971-1996) 65 (1981) 316.

[19] M. Y. Khlopov, C. A. Stephan and D. Fargion, Dark matter with invisible light from heavy double charged leptons of almost-commutative geometry?, Classical and Quantum Gravity 23 (2006) 7305.

[20] C. Mascaretti and F. Vissani, On the relevance of prompt neutrinos for the interpretation of the icecube signals, Journal of Cosmology and Astroparticle Physics 2019 (2019) 004.

[21] N. Wandkowsky, Latest results on astrophysical neutrinos using high-energy events with contained vertices, in Poster at XXVIII International Conference on Neutrino Physics and Astrophysics, 4-9 June 2018, Heidelberg, Germany, Zenodo, 2018, DOI. 
[22] I. Taboada, A view of the universe with the icecube and antares neutrino telescopes, in Talk at XXVIII International Conference on Neutrino Physics and Astrophysics, 4-9 June 2018, Heidelberg, Germany, Zenodo, 2018, DOI.

[23] M. Usner, Search for Astrophysical Tau-Neutrinos in Six Years of High-Energy Starting Events in the IceCube Detector, Ph.D. thesis, Humboldt-Universitat zu Berlin, Mathematisch-Naturwissenschaftliche Fakultat, 2018. http://dx.doi.org/10.18452/19458.

[24] J. G. Learned and S. Pakvasa, Detecting tau-neutrino oscillations at PeV energies, Astropart. Phys. 3 (1995) 267 [hep-ph/9405296].

[25] R. Enberg, M. H. Reno and I. Sarcevic, Prompt neutrino fluxes from atmospheric charm, Phys. Rev. D78 (2008) 043005 [0806.0418].

[26] D. Fargion, Discovering Ultra High Energy Neutrinos by Horizontal and Upward tau Air-Showers: Evidences in Terrestrial Gamma Flashes?, Astrophys. J. 570 (2002) 909 [astro-ph/ 0002453 ].

[27] D. Fargion, P. G. De Sanctis Lucentini and M. De Santis, Tau air showers from earth, Astrophys. J. 613 (2004) 1285 [hep-ph/ 0305128$].$

[28] D. Fargion, A. Aiello and R. Conversano, Horizontal tau air showers from mountains in deep valley: Traces of UHECR neutrino tau, in Proceedings, 26th International Cosmic Ray Conference (ICRC), August 17-25, 1999, Salt Lake City: Invited, Rapporteur, and Highlight Papers, p. 396, 1999, astro-ph/9906450.

[29] J. L. Feng, P. Fisher, F. Wilczek and T. M. Yu, Observability of earth-skimming ultrahigh energy neutrinos, Physical Review Letters 88 (2002).

[30] M. Usner, I. Collaboration et al., Search for astrophysical tau neutrinos in six years of high-energy starting events in icecube, in 35th International Cosmic Ray Conference, vol. 301, p. 974, SISSA Medialab, 2018, DOI.

\section{DISCUSSION}

ULI KATZ: The events observed by ICECUBE are not consistent with atmospheric prompt neutrinos since these could be accompanied by atmospheric muons that are filtered out by HESE veto.

DANIELE FARGION: It is true that prompt charmed neutrinos might come with their twin muon companion, but only in less than half of the downward muon neutrinos sky. Horizontal and Upward muon neutrinos are not coming by sure with any muon (absorbed) anyway. Now consider the following question: Why highest energetic $(>90) \mathrm{TeV}$ downward muon tracks are also almost absent (two events versus 24 showers) while they are well observed ( 6 tracks versus 10 showers) in upward sky? The answer coulb be because most of them are not of astrophysical nature, but they are charmed ones and excluded and filtered by ICECUBE veto. Moreover, there is wide agreement in different articles that the atmospheric expected prompt flux is not well defined up to a factor of two. Therefore, prompt neutrino may well explain several puzzles at once. 
ULI KATZ: The numbers of good tau neutrinos candidates passing the corresponding event selection is expected to be small, about two events for the full data sample. It is therefore, much too early to draw conclusions from the fact that no such events are observed so far.

DANIELE FARGION: I agree that the expected two events (since June 2018, possibly observed and reported in Neutrino 2018) within nine events above $200 \mathrm{TeV}$ might be consistent in a first view with the expected ones. However, the same June 2018 Neutrino report informed us that there are many more (27) events candidate above $100 \mathrm{TeV}$ that are in principle showing a double bang; they are possibly nine tau ones. Their low number (or absence) is quite surprising. More new recent suppression efficiency in tau detection may offer the escape road to the puzzle. Anyway the atmospheric charmed tau would be, within 34 showers above $90 \mathrm{TeV}$ energy, anyway about one or two events. Therefore, we believe that the missing tau is still a persistent puzzle, finding a natural solution in a dominant charmed component. 